\title{
Photocathode device using diamondoid and cesium bromide films
}

\author{
William A. Clay, ${ }^{1}$ Juan R. Maldonado, ${ }^{2}$ Piero Pianetta, ${ }^{2}$ Jeremy E. P. Dahl, ${ }^{3}$ \\ Robert M. K. Carlson, ${ }^{3}$ Peter R. Schreiner, ${ }^{4}$ Andrey A. Fokin, ${ }^{4}$ \\ Boryslav A. Tkachenko, ${ }^{4}$ Nicholas A. Melosh, ${ }^{5}$ and Zhi-Xun Shen ${ }^{1,2}$ \\ ${ }^{1}$ Department of Physics, Stanford University, Stanford, California 94305, USA \\ ${ }^{2}$ Stanford Synchrotron Radiation Laboratory, Stanford University, Menlo Park, California 94309, USA \\ ${ }^{3}$ Stanford Institute of Materials and Energy Science, Stanford University, Stanford, California 95305, USA \\ ${ }^{4}$ Institute of Organic Chemistry, Justus-Liebig-University, Heinrich-Buff-Ring 58, D-35392 Giessen, Germany \\ ${ }^{5}$ Department of Materials Science, Stanford University, Stanford, California 94305, USA
}

(Received 7 July 2012; accepted 12 November 2012; published online 12 December 2012)

\begin{abstract}
A photocathode structure is presented that shows promise for use in high brightness electron sources. The structure consists of a metal substrate, a monolayer of a diamondoid derivative, and a thin film of cesium bromide. Diamondoid monolayers reduce the energy spread of electron emitters, while cesium bromide increases the yield and stability of cathodes. We demonstrate that the combined structure retains these properties, producing an emitter with lower energy spread than the corresponding cesium bromide emitter $(1.06 \mathrm{eV}$ versus $1.45 \mathrm{eV})$ and higher yield and stability than un-coated diamondoid emitters. (C) 2012 American Institute of Physics. [http://dx.doi.org/10.1063/1.4769043]
\end{abstract}

High brightness photocathodes are desirable for many applications, including electron beam lithography, ${ }^{1}$ electron microscopes, ${ }^{2}$ and free electron lasers (FEL). ${ }^{3}$ An ideal emitter for these devices would have high intensity, small physical size, good stability under operating conditions, and low energy spread. One method for achieving high yield and small physical size is to use a focused laser beam to create photoelectrons. However, performance of such devices is limited by the quantum yield and the energy spread. In this work, we present a photocathode structure that improves on the quantum yield and reduces the energy spread of laser photoemission devices without sacrificing the other desirable properties. This device consists of a monolayer of diamondoid combined with a thin film of cesium bromide.

Diamondoids are hydrocarbon molecules with the same carbon-lattice structure as bulk diamond. They inherit many of the superior properties of diamond ${ }^{4,5}$ and possess emergent properties relating to their small size and high surface area ratio. ${ }^{6-12}$ One unique property of these diamondoids is their behavior in photoemission devices. A monolayer of diamondoids on a metal substrate can largely monochromatize the photoemission, with the majority of the electrons emitting in a single peak with a full-width half-max (FWHM) of around $0.3 \mathrm{eV} .^{6,7}$ This low energy spread is ideal for many cathode applications, but the diamondoid monolayers that have been studied are not stable enough for use in most devices. In order to correct this problem, we have added cesium bromide as a protective over-layer to provide mechanical stability and protection.

Cesium bromide provides more than just protection, however. $\mathrm{CsBr}$ coatings on photocathodes have been extensively studied $^{13-18}$ and have desirable properties. They greatly enhance the quantum yield of the emitter by reducing the effective work function and are robust and capable of operation under the demanding conditions of ultra bright FEL sources. ${ }^{19}$ Our objective is to combine these attractive properties with the reduced energy spread of diamondoids by creating a device that contains both films using a thiolated diamondoid to create a self-assembled monolayer (SAM).
We tested these devices using ultra-violet (UV) laser photoemission, as shown in Figure 1. We measured the quantum yield, the energy spread, and lifetime of devices with and without the diamondoid present with varying thicknesses of CsBr. Through these investigations, we demonstrate that the high quantum yield of the $\mathrm{CsBr}$ emitter is preserved when the diamondoid is added and that the lifetime of the emitter is enhanced five times relative to that of the unprotected diamondoid. Additionally, we find that very thin films of $\mathrm{CsBr}$ deposited over diamondoids produce a significant reduction of energy spread relative to $\mathrm{CsBr}$ only emitters, with the energy spread being reduced from $1.45 \mathrm{eV}$ FWHM to $1.06 \mathrm{eV}$ FWHM for a $10 \AA \mathrm{CsBr}$ film on gold.

While the $1.06 \mathrm{eV}$ FWHM energy spread is a significant improvement, it is not as good as the $0.38 \mathrm{eV}$ FWHM measured for diamondoid without $\mathrm{CsBr}$. There are several possible causes for this, which we will discuss further. Nonetheless, the device has succeeded in reducing the energy spread of a $\mathrm{CsBr}$ photocathode while maintaining its other superior properties.

The mechanism of photoemission enhancement has been studied for both diamondoids ${ }^{7,20}$ and $\mathrm{CsBr} .{ }^{15,16}$ For diamondoids, the monolayer acts as an energy filter. The diamondoids exhibit an unusually short mean free path (MFP) and negative electron affinity (NEA). The short MFP causes electrons to quickly accumulate in the lowest unoccupied states, and the NEA allows electrons in this state to be emitted into vacuum, producing a high intensity sharp emission peak.

$\mathrm{CsBr}$, on the other hand, provides protection and work function reduction. As a protective layer, it is a stable film that can be easily regenerated if damaged. It is also fairly transparent to low energy electrons due to its large band gap. The mechanism for work function reduction requires the generation of color center defects in the film; defect free $\mathrm{CsBr}$ is a good insulator and would not have a strong electronic interaction with the gold. If certain color centers are created, however, they form defect states within the band gap. Because the $\mathrm{CsBr}$ is in good contact with the gold, these defect states become "pinned" to the gold Fermi level. This, combined with a surface monolayer of cesium, produces a 


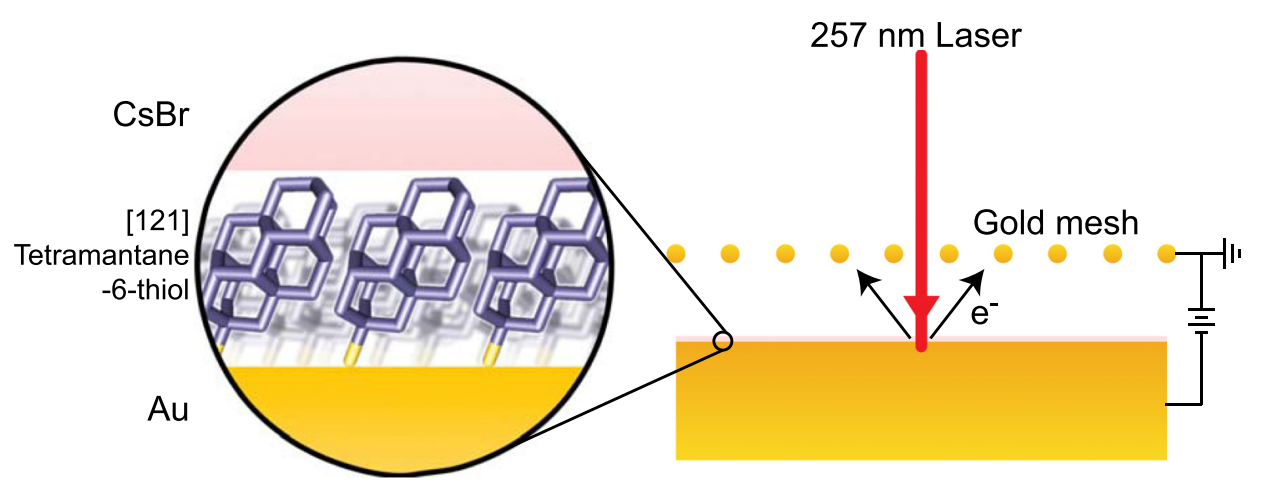

FIG. 1. Schematic diagram of Au/diamondoid/CsBr photoemission device and measurement setup. Expanded view on left shows internal structure of the film consisting of a monolayer of [121]tetramantane-6thiol (hydrogens not shown for clarity) and a thin film of $\mathrm{CsBr}$. On right, the geometry of the measurement is shown with the laser passing through a wire mesh and hitting the sample to excite photoelectrons that are captured on the mesh.

large dipole moment that reduces the work function. Because the work function determines the energy barrier for photoemission, reducing the work function can vastly increase the photo yield. These defects can be produced through laser irradiation.

The mechanism for the composite device is a simple combination of the mechanism of each component. The diamondoid layer acts as a filter to produce monochromatic electrons, and the $\mathrm{CsBr}$ acts as a protective, electron transparent layer that reduces the work function. This produces an emitter with the best properties of both systems.

Single crystal copper substrates were obtained from MTI Corporation in Richmond, CA and were used as received. These were selected for their high thermal conductivity and because they are extremely smooth $(<10 \AA \mathrm{rms}$ roughness). These wafers were coated with $5 \mathrm{~nm}$ of titanium and $100 \mathrm{~nm}$ of gold using a magnetron sputtering system. Gold was used for consistency with prior diamondoid work. ${ }^{6,20}$

Diamondoids were isolated using methods described elsewhere. ${ }^{11,12}$ They were functionalized with thiol groups to allow attachment to gold using methods described previously. $^{21,22}$ [121]Tetramantane-6-thiol was used in order to compare with previous results. These diamondoid-thiols were attached to the gold using self-assembly methods, which have been described elsewhere. 6,23,24

Experiments were performed at the Source Development Test stand (SDT) ${ }^{15}$ at the Stanford Synchrotron Radiation Laboratory (SSRL), which contains a UHV system with a load lock chamber, a sample prep chamber, and an analysis chamber. For substrates without diamondoid, the load lock was heated to a temperature of at least $100{ }^{\circ} \mathrm{C}$ to decrease the required pumping time. This was not done for the diamondoidthiol films because they are not stable at that temperature, so the diamondoid samples were left in the load lock for three days to reach a sufficiently low pressure. Previous work has shown that the heating process does not affect the performance of the cathodes. ${ }^{13}$

Cesium bromide was obtained from Alfa Aesar and was used as received. It was sublimated onto the substrates using a thermal effusion cell with a BN crucible heated to $418^{\circ} \mathrm{C}$.

Photoemission was measured in the analysis chamber at a pressure of $5 \times 10^{-10}$ Torr or lower. Photoemission was excited with an Innova 300C FreD argon gas frequency doubled laser. The laser light had a wavelength of $257 \mathrm{~nm}$ and the spot was approximately $1 \mathrm{~mm}$ diameter. The laser power on the sample was approximately $14 \mathrm{~mW}$ for lifetime and quantum yield measurements. For energy spread meas- urements, the power was reduced by up to $90 \%$ in order to reduce the photocurrent to $120 \mathrm{nA}$. This was done to minimize the effects of sample charging and contact resistance and ensure that all samples were measured at the same current.

Photocurrent was measured using a gold mesh $2 \mathrm{~mm}$ above the sample, as shown in Figure 1. The mesh was grounded to the chamber walls and the sample was biased using a battery. For the quantum yield and lifetime measurements, the sample was biased to $-300 \mathrm{~V}$ to ensure collection of all photoelectrons. For the energy spread measurements, the sample was biased using a $36 \mathrm{~V}$ battery supply connected to a voltage divider that allowed the voltage to be continuously adjusted over the range from $-18 \mathrm{~V}$ to $+18 \mathrm{~V}$. The energy spread was measured by sweeping the retarding voltage.

Prior to measurement, all samples were treated under UV laser irradiation until the yield reached a peak and began to decline. For samples with diamondoids but no $\mathrm{CsBr}$, this was done to clean the sample. For $\mathrm{CsBr}$ samples, this was done to activate the film by creating color centers through radiation damage.

The quantum yield was determined by comparing the laser power on the sample to the maximum photocurrent. For the $\mathrm{Au}$ /diamondoid films without $\mathrm{CsBr}$, the yield was $9 \mathrm{nW} /$ $\mathrm{mW}$. For the $\mathrm{Au} / \mathrm{diamondoid} / \mathrm{CsBr}$ samples, it was between $107 \mathrm{nA} / \mathrm{mW}$ and $123 \mathrm{nA} / \mathrm{mW}$. For the $\mathrm{Au} / \mathrm{CsBr}$ samples without diamondoid, it was between $106 \mathrm{nA} / \mathrm{mW}$ and $136 \mathrm{nA} / \mathrm{mW}$. Due to the uncertainty in the power measurement and variations between spots on the same sample, the error on these measurements is around $\pm 10 \%$.

The energy spread was measured by sweeping the retarding voltage between the photocathode and the mesh anode. Only electrons with enough kinetic energy to overcome the retarding voltage and the work function difference between the cathode and anode will reach the anode and be collected. Because the work function difference is constant, the current-voltage (I/V) curve should give the integral of the energy distribution as a function of energy, and the derivative $(\mathrm{dI} / \mathrm{dV})$ curve should give the energy distribution directly.

Due to noise in the data that can be amplified when taking derivatives, we chose to analyze the I/V curve directly. We assumed that the energy distribution could be approximated by a Gaussian so the analysis was done by fitting the $\mathrm{I} / \mathrm{V}$ curve to the integral of a Gaussian, known as the error function. The fitting equation was 


$$
I(V)=C_{0}+C_{1} \int_{-\infty}^{V} \exp \left(\frac{\left(V^{\prime}-V_{c}\right)^{2}}{2 \sigma^{2}}\right) d V^{\prime}
$$

where $C_{0}$ are $C_{1}$ are arbitrary constants, $V_{c}$ is the center of the distribution, and $\sigma$ is the width. The reported FWHM was calculated as $2.3548 \sigma$. Examples of this fitting are shown in Figure 2, which shows one fit for a $\mathrm{Au} / \mathrm{CsBr}$ sample and one for a $\mathrm{Au} /$ diamondoid/CsBr sample. Also plotted are the $\mathrm{dI} / \mathrm{dV}$ data compared with a Gaussian function computed from the error function fit. For both cases, the error function tracks the raw I/V data well and the corresponding Gaussian matches reasonably with the $\mathrm{dI} / \mathrm{dV}$ data.

Using this technique, we determined the energy spread as a function of the $\mathrm{CsBr}$ thickness. For the $\mathrm{Au} / \mathrm{CsBr}$ samples without diamondoid, we found that the FWHM was nearly constant at all $\mathrm{CsBr}$ thicknesses and the average was 1.44 $\pm 0.05 \mathrm{eV}$ FWHM. For the Au/diamondoid/CsBr samples,

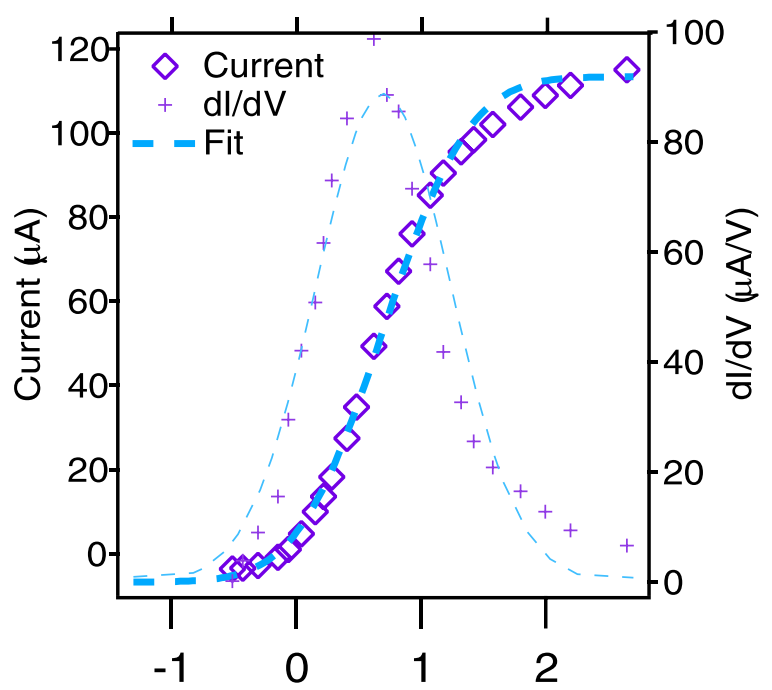

\section{Cathode-Anode Voltage (V)}

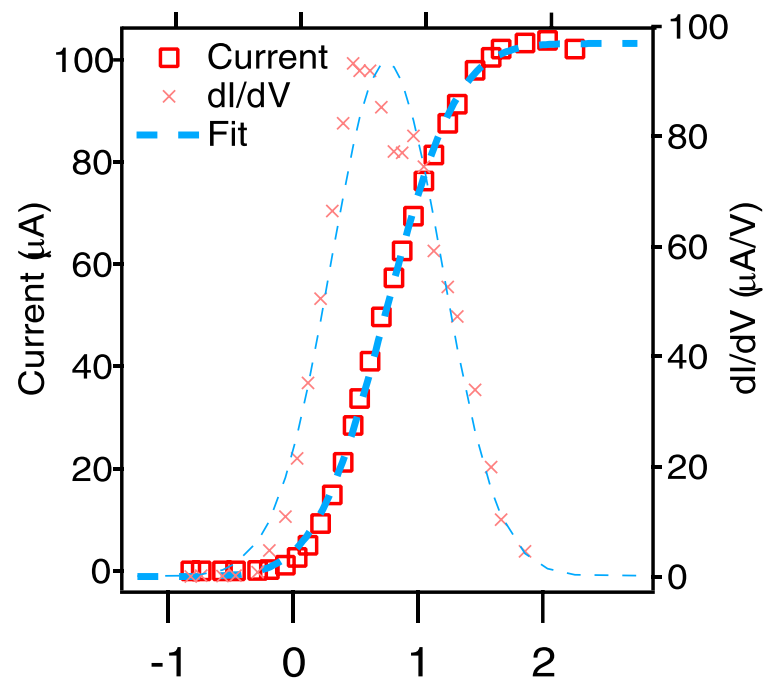

Cathode-Anode Voltage (V)

FIG. 2. I/V curves for several samples showing the curve fitting results. The top panel shows a $\mathrm{Au} / \mathrm{CsBr}$ sample with no diamondoid, while the bottom shows a $\mathrm{Au} / \mathrm{diamondoid} / \mathrm{CsBr}$ sample. The thick dotted line shows the error function fit to the data. Also shown is the $\mathrm{dI} / \mathrm{dV}$ data with the Gaussian function corresponding to the fit (thin dotted line). however, we observed a strong dependence on the FWHM on the thickness. The full results for all Au/diamondoid/ $\mathrm{CsBr}$ samples are shown in Figure 3 with a line showing the value for the $\mathrm{Au} / \mathrm{CsBr}$ samples for comparison.

As an initial comment on the energy spread data, we note that the measured value for the $\mathrm{Au} /$ diamondoid film without $\mathrm{CsBr}$ of $0.38 \pm 0.08 \mathrm{eV}$ is in excellent agreement with the published value of $0.3 \mathrm{eV}$ measured using a high-resolution electron analyzer. ${ }^{7}$ This indicates that our resolution is high enough for this measurement, probably between 0.1 and $0.2 \mathrm{eV}$. The measurement of the diamondoid sample without $\mathrm{CsBr}$ also confirmed the presence of diamondoids, as bare gold will not photoemit at all under $257 \mathrm{~nm}$ light because the photon energy is less than gold's work function. ${ }^{25}$

We note that there is initially a strong relation between the CsBr thickness and the FWHM which quickly levels out above $25 \AA$ and approaches a value very near the nodiamondoid FWHM. There are several possible explanations for this behavior. One is that $\mathrm{CsBr}$ actively increases the energy spread either by scattering the electrons or producing its own photoelectrons with a higher energy spread. However, $\mathrm{CsBr}$ films on other materials do not produce the same effect. $\mathrm{CsBr}$ on $\mathrm{InGaN}$, for example, produces an energy spread less than $0.5 \mathrm{eV}$ FWHM with $\mathrm{CsBr}$ thicknesses well above $100 \AA$ (Ref. 17) in measurements with the sample illuminated from the rear, and $\mathrm{CsBr}$ on copper substrates produce a FWHM of $0.77 \mathrm{eV}$ in measurements performed using the same equipment and technique as this work. ${ }^{18}$ Therefore, it seems unlikely that $\mathrm{CsBr}$ directly increases the energy spread.

A more plausible explanation is that $\mathrm{CsBr}$ does not diminish performance but the diamondoid film is damaged during $\mathrm{CsBr}$ evaporation. If the fractional rate of damage is constant in time, this would produce an exponential decay curve, which we have fit to the data in Figure 3. The CsBr is heated to a high temperature $418^{\circ} \mathrm{C}$ but the diamondoid films cannot survive temperatures above $60^{\circ} \mathrm{C}$, so it is

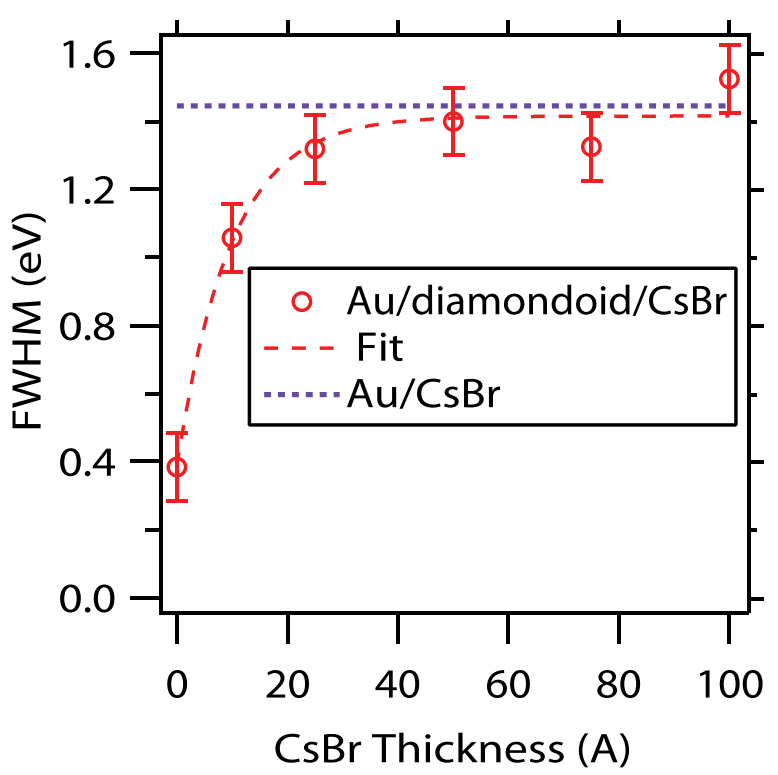

FIG. 3. FWHM as a function of thickness for Au/diamondoid/CsBr samples. FWHM calculated from fit. Also shown are an exponential fit to the data (dotted line) and the FWHM for $\mathrm{Au} / \mathrm{CsBr}$ samples (horizontal line). 
plausible that hot $\mathrm{CsBr}$ heats the substrate enough to damage the film. We cannot directly confirm this, however, because we have no means of detecting the diamondoid in-situ once the $\mathrm{CsBr}$ is added and the film would not survive removal into air. This damage problem could likely be reduced by optimizing the growth conditions by modifying substrate temperature, evaporation rate, or evaporation technique.

There are other possible mechanisms for the energy spread behavior that cannot be entirely ruled out. One is that there may be complex interface interactions between the diamondoid and the $\mathrm{CsBr}$. The $\mathrm{CsBr}$ is highly polar at the surface and will likely not bond as strongly to the insulating diamondoid layer as the underlying gold layer, which may produce unpredictable effects on electrons transiting between the two layers. Because we cannot confirm the damage mechanism, we cannot rule out any other possible mechanism completely.

Despite these limitations, we find that a film of diamondoid beneath $\mathrm{CsBr}$ produces a marked reduction in the FWHM, reducing it from $1.44 \pm 0.04$ to $1.06 \pm 0.1 \mathrm{eV}$ for $10 \AA \mathrm{CsBr}$ films. The yield of the $10 \AA$ sample was $107 \mathrm{nA} /$ $\mathrm{mW}$, which is similar to most of the films that had no diamondoid. Thus, we see that we are able to considerably reduce the energy spread of the cathode while maintaining the yield, producing a clear improvement in the quality of the emitter.

The final property measured was the lifetime. This was done by illuminating the sample at full power until it reached its peak yield and then observing the yield as a function of time. The results of this measurement for a Au/diamondoid/ $\mathrm{CsBr}(10 \AA)$ sample and a $\mathrm{Au} /$ diamondoid sample are shown in Figure 4. In the figure, we note that there is a gap in the middle of the $\mathrm{Au} /$ diamondoid/CsBr data; this corresponded to an energy spread measurement. The sample was illuminated for this entire time but the current is not included here because the voltage was being adjusted for the energy spread measurement.

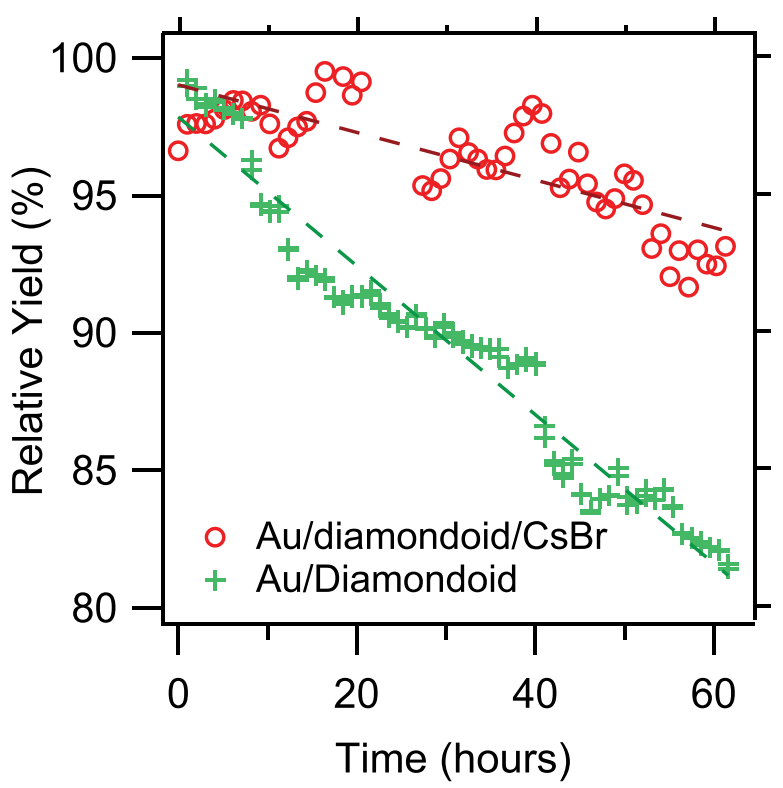

FIG. 4. Relative photoyield for Au/diamondoid (green crosses) and Au/diamondoid/CsBr (red circles) with linear fits.
In addition to the raw data, we have added linear fits which were used to calculate the decay rate. Over the course of the $62 \mathrm{~h}$, the $\mathrm{Au} / \mathrm{diamondoid} / \mathrm{CsBr}$ film loses about $3 \%$ of its yield, a rate of just $1.2 \%$ per day. The Au/diamondoid film loses roughly $16 \%$ of its yield, a rate of $6.2 \%$ per day. Thus, we see that the addition of the $\mathrm{CsBr}$ layer over the top of the diamondoid layer enhances the lifetime of the diamondoid monolayer by more than a factor of five. To ensure that the diamondoid layer is still effective at reducing the energy spread at the end of the lifetime measurement, we remeasured the FWHM and found it to be $1.07 \pm 0.1 \mathrm{eV}$, nearly the same as it had been in previous measurements.

Despite the considerable improvement in the energy spread, the final value of $1.06 \pm 0.1 \mathrm{eV}$ is still too large for many applications and is indeed higher than the measured energy spread of $\mathrm{CsBr}$ films deposited directly onto certain other materials such as InGaN and copper metal. ${ }^{17,18}$ However, we have demonstrated the ability of diamondoids to reduce the energy spread of these types of devices, and it should be possible to add the diamondoid monolayers onto the other, more desirable substrates if a suitable monolayer attachment method can be found.

In summary, we have developed a thin film structure for use in photocathode devices by combining a diamondoid monolayer with a thin film of $\mathrm{CsBr}$ and tested this architecture using diamondoid-thiol SAMs. We have tested the yield, the energy spread, and the lifetime of these devices and found that they maintain the attractive properties of $\mathrm{CsBr}$ cathodes, namely high yield and good stability, while improving the energy spread. These results are a promising demonstration of the properties of diamondoid/CsBr photocathodes and indicate that these materials could be used in a superior photocathode device with many potential applications.

This work was supported, in part, by a grant through the SLAC National Accelerator Laboratory (SNAL) Laboratory Discretionary Research Directorate (LDRD), as well as by the DOE Office of Basic Energy Science, Division of Material Science and Engineering under Contract No. DE-AC0276 SF00515.

${ }^{1}$ S. T. Coyle, B. Shamoun, M. Yu, J. Maldonado, T. Thomas, D. Holmgren, X. Chen, M. R. Scheinfein, B. DeVore, and M. Gesley, J. Vac. Sci. Technol. B 22, 501 (2004).

${ }^{2}$ D. S. Pickard, C. Campbell, T. Crane, L. J. Cruz-Rivera, A. Davenport, W. D. Meisburger, R. F. W. Pease, and T. R. Groves, J. Vac. Sci. Technol. B 20, 2662 (2002).

${ }^{3}$ R. Tatchyn, J. Arthur, M. Baltay, K. Bane, R. Boyce, M. Cornacchia, T. Cremer, A. Fisher, S.-J. Hahn, M. Hernandez, G. Loew, R. Miller, W. R. Nelson, H.-D. Nuhn, D. Palmer, J. Paterson, T. Raubenheimer, J. Weaver, H. Wiedemann, H. Winick, C. Pellegrini, G. Travish, E. T. Scharlemann, S. Caspi, W. Fawley, K. Halbach, K.-J. Kim, R. Schlueter, M. Xie, D. Meyerhofer, R. Bonifacio, and L. De Salvo, Nucl. Instrum. Methods Phys. Res. A 375, 274 (1996).

${ }^{4}$ T. M. Willey, C. Bostedt, T. van Buuren, J. E. Dahl, S. G. Liu, R. M. K. Carlson, L. J. Terminello, and T. Moller, Phys. Rev. Lett. 95, 113401 (2005).

${ }^{5}$ H. Schwertfeger, A. A. Fokin, and P. R. Schreiner, Angew. Chem., Int. Ed. 47, 1022 (2008).

${ }^{6}$ W. L. Yang, J. D. Fabbri, T. M. Willey, J. R. I. Lee, J. E. Dahl, R. M. K. Carlson, P. R. Schreiner, A. A. Fokin, B. A. Tkachenko, N. A. Fokina, W. Meevasana, N. Mannella, K. Tanaka, X. J. Zhou, T. van Buuren, M. A. Kelly, Z. Hussain, N. A. Melosh, and Z.-X. Shen, Science 316, 1460 (2007). 
${ }^{7}$ W. A. Clay, Z. Liu, W. L. Yang, J. D. Fabbri, J. E. P. Dahl, R. M. K. Carlson, Y. Sun, P. R. Schreiner, A. A. Fokin, B. A. Tkachenko, N. A. Fokina, P. A. Pianetta, N. A. Melosh, and Z.-X. Shen, Nano Lett. 9, 57 (2009).

${ }^{8}$ W. Meevasana, R. Supruangnet, H. Nakajima, O. Topon, V. Amornkitbamrung, and P. Songsiriritthigul, Appl. Surf. Sci. 256, 934 (2009).

${ }^{9}$ W. A. Clay, T. Sasagawa, A. Iwasa, Z. Liu, J. E. Dahl, R. M. K. Carlson, M. Kelly, N. A. Melosh, and Z.-X. Shen, J. Appl. Phys. 110, 093512 (2011).

${ }^{10}$ W. A. Clay, T. Sasagawa, M. Kelly, J. E. Dahl, R. M. K. Carlson, N. A. Melosh, and Z.-X. Shen, Appl. Phys. Lett. 93, 172901 (2008).

${ }^{11}$ J. E. P. Dahl, S. G. Liu, and R. M. K. Carlson, Science 299, 96 (2003).

${ }^{12}$ J. E. P. Dahl, J. M. Moldowan, T. M. Peakman, J. C. Clardy, E. Lobkovsky, M. M. Olmstead, P. W. May, T. J. Davis, J. W. Steeds, K. E. Peters, A. Pepper, A. Ekua, and R. M. K. Carlson, Angew. Chem., Int. Ed. 42, 2040 (2003).

${ }^{13}$ J. R. Maldonado, Z. Liu, D. H. Dowell, R. E. Kirby, Y. Sun, P. Pianetta, and F. Pease, Microelectron. Eng. 86, 529 (2009)

${ }^{14}$ J. R. Maldonado, S. T. Coyle, B. Shamoun, M. Yu, M. Gesley, and P. Pianetta, J. Vac. Sci. Technol. B 22, 3025 (2004).

${ }^{15}$ J. R. Maldonado, Z. Liu, Y. Sun, P. A. Pianetta, and F. W. Pease, J. Vac. Sci. Technol. B 24, 2886 (2006).

${ }^{16}$ Z. Liu, J. Maldonado, Y. Sun, P. Pianetta, and R. F. W. Pease, Appl. Phys. Lett. 89, 111114 (2006).
${ }^{17}$ J. R. Maldonado, Y. Sun, Z. Liu, X. Liu, S. Tanimoto, P. Pianetta, and F. Pease, J. Vac. Sci. Technol. B 26, 2085 (2008).

${ }^{18}$ J. R. Maldonado, P. Pianetta, D. H. Dowell, J. Corbett, S. Park, J. Schmerge, A. Trautwein, and W. Clay, Appl. Phys. Lett. 101(23), 231103 (2012).

${ }^{19}$ J. R. Maldonado, Z. Liu, D. H. Dowell, R. E. Kirby, Y. Sun, P. Pianetta, and F. Pease, Phys. Rev. ST Accel. Beams 11, 060702 (2008).

${ }^{20}$ S. Roth, D. Leuenberger, J. Osterwalder, J. E. P. Dahl, R. M. K. Carlson, B. A. Tkachenko, A. A. Fokin, P. R. Schreiner, and M. Hengsberger, Chem. Phys. Lett. 495, 102 (2010).

${ }^{21}$ P. R. Schreiner, N. A. Fokina, B. A. Tkachenko, H. Hausmann, M. Serafin, J. E. P. Dahl, S. G. Liu, R. M. K. Carlson, and A. A. Fokin, J. Org. Chem. 71, 6709 (2006).

${ }^{22}$ B. A. Tkachenko, N. A. Fokina, L. V. Chernish, J. E. P. Dahl, S. G. Liu, R. M. K. Carlson, A. A. Fokin, and P. R. Schreiner, Org. Lett. 8, 1767 (2006).

${ }^{23}$ A. Ulman, Chem. Rev. 96, 1533 (1996).

${ }^{24}$ T. M. Willey, C. Bostedt, T. van Buuren, J. E. Dahl, S. G. Liu, R. M. K. Carlson, R. W. Meulenberg, E. J. Nelson, and L. J. Terminello, Phys. Rev. B 74, 205432 (2006).

${ }^{25}$ CRC Handbook of Chemistry and Physics, edited by D. R. Lide, 88th ed. (CRC/Taylor and Francis, Boca Raton, FL, 2008) 